\title{
Field-aligned chorus wave spectral power in Earth's outer radiation belt
}

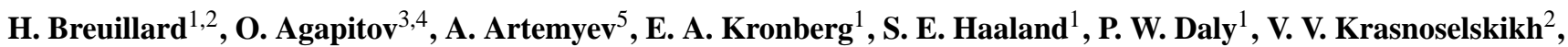 \\ D. Boscher ${ }^{6}$, S. Bourdarie ${ }^{6}$, Y. Zaliznyak ${ }^{7}$, and G. Rolland ${ }^{8}$ \\ ${ }^{1}$ Max-Planck Institut für Sonnensystemforschung, Göttingen, Germany \\ ${ }^{2}$ LPC2E/CNRS-University of Orléans, Orléans, France \\ ${ }^{3}$ Space Sciences Laboratory, University of California, Berkeley, USA \\ ${ }^{4}$ National Taras Shevchenko University of Kyiv, Kyiv, Ukraine \\ ${ }^{5}$ Space Research Institute, RAS, Moscow, Russia \\ ${ }^{6}$ ONERA the French Aerospace Lab, Toulouse, France \\ ${ }^{7}$ Institute for Nuclear Research, Kyiv, Ukraine \\ ${ }^{8} \mathrm{CNES}$, Toulouse, France \\ Correspondence to: H. Breuillard (hbreuill@gmail.com)
}

Received: 28 January 2015 - Revised: 17 April 2015 - Accepted: 30 April 2015 - Published: 29 May 2015

\begin{abstract}
Chorus-type whistler waves are one of the most intense electromagnetic waves generated naturally in the magnetosphere. These waves have a substantial impact on the radiation belt dynamics as they are thought to contribute to electron acceleration and losses into the ionosphere through resonant wave-particle interaction. Our study is devoted to the determination of chorus wave power distribution on frequency in a wide range of magnetic latitudes, from 0 to $40^{\circ}$. We use 10 years of magnetic and electric field wave power measured by STAFF-SA onboard Cluster spacecraft to model the initial (equatorial) chorus wave spectral power, as well as PEACE and RAPID measurements to model the properties of energetic electrons $(\sim 0.1-100 \mathrm{keV})$ in the outer radiation belt. The dependence of this distribution upon latitude obtained from Cluster STAFF-SA is then consistently reproduced along a certain $L$-shell range ( $4 \leq L \leq 6.5$ ), employing WHAMP-based ray tracing simulations in hot plasma within a realistic inner magnetospheric model. We show here that, as latitude increases, the chorus peak frequency is globally shifted towards lower frequencies. Making use of our simulations, the peak frequency variations can be explained mostly in terms of wave damping and amplification, but also cross- $L$ propagation. These results are in good agreement with previous studies of chorus wave spectral extent using data from different spacecraft (Cluster, POLAR and THEMIS). The chorus peak frequency variations are then
\end{abstract}

employed to calculate the pitch angle and energy diffusion rates, resulting in more effective pitch angle electron scattering (electron lifetime is halved) but less effective acceleration. These peak frequency parameters can thus be used to improve the accuracy of diffusion coefficient calculations.

Keywords. Electromagnetics (wave propagation) - magnetospheric physics (energetic particles precipitating) - space plasma physics (wave-particle interactions)

\section{Introduction}

The assessment of radiation belt dynamics is one of the most important objectives of space weather programmes (Baker, 2002), because of the impact of energetic particles on technology (e.g. Wrenn et al., 2002; Daglis et al., 2004; Iucci et al., 2005) and Earth's environment (e.g. Thorne, 1977; Lanzerotti, 2012). Energetic electrons are thought to be accelerated (up to $\sim \mathrm{MeV}$ energies) and lost into the atmosphere to a large extent by resonant wave-particle interactions (see the comprehensive review by Thorne, 2010), especially during high geomagnetic activity (see Horne, 2002; Chen et al., 2007; Shprits et al., 2008).

To define the dynamics of radiation belts, the timescale for electron loss and acceleration is calculated by numerical 
models (Bourdarie et al., 1996; Li et al., 2001; Glauert and Horne, 2005; Summers et al., 2007; Fok et al., 2008; Mourenas et al., 2012a, b, 2014), that are based on the quasi-linear theory. Using this approach, in which resonant wave-particle interactions are described in terms of particle pitch angle and energy diffusion (Kennel and Petschek, 1966; Trakhtengertz, 1966; Lyons and Williams, 1984), it has been shown that chorus-type whistler waves play a major role in both the loss and local acceleration of radiation belt electrons (see e.g. Bortnik and Thorne, 2007; Summers et al., 2007; Shprits et al., 2008; Santolík et al., 2010; Thorne, 2010, and references therein).

Realistic distributions of chorus wave power in the radiation belts should thus be included in diffusion models to accurately reproduce the effects of wave-particle interactions on energetic electrons (Summers, 2005; Shprits et al., 2006; Albert, 2007), in particular the latitudinal distributions on which diffusion rates can depend sensitively (Summers and $\mathrm{Ni}, 2008$ ). In these models, the diffusion rates are determined in terms of plasma and magnetic field parameters, wave power distribution upon amplitude, frequency and wave-normal angle propagation (see e.g. Lyons, 1974; Glauert and Horne, 2005; Artemyev et al., 2012a). Because of the large latitudinal extent of chorus wave power in the day side (e.g. Bunch et al., 2012; Tsurutani et al., 2012; Agapitov et al., 2013), parameters of chorus wave distributions have been determined recently along magnetic field lines up to high latitudes. These parameters have made it possible to estimate the effects of the latitudinal dependence of chorus wave-normal angle and amplitude on the electron diffusion rates. However, observed field-aligned dependence of chorus spectral parameters have not been provided nor explained yet. These parameters would enable the accurate calculation of chorus spectral extent impact on the outer radiation belt dynamics, notably at high latitudes (Bunch et al., 2013).

Bunch et al. (2013) have used observations carried out on POLAR spacecraft to determine the off-equatorial chorus spectral extent for different $L$-shells and latitude ranges, and to deduce first estimations of their impact on diffusion rates. However, due to the POLAR orbit and the time range of the statistics used, the full range of chorus latitudinal extent could not be covered for each specific $L$-shell. The results obtained in Bunch et al. (2013) overall agree with previous results obtained (e.g. Burtis and Helliwell, 1969; Ni et al., 2011) showing the decrease of peak frequency as latitude increases along a specific $L$, but hint an inflection at about $25^{\circ}$ latitude which cannot be explained by the ray tracing calculations in Burtis and Helliwell (1969). In the latter paper, only the decrease of the peak frequency with increasing latitude has been observed, and interpreted as the inward propagation of waves from higher $L$, thus with a lower frequency. Bunch et al. (2013) concluded that the inflection observed on POLAR is likely the result of competing effects of wave growth/damping and cross- $L$ propagation, and that an extensive ray tracing study is needed to sort out the different factors.

In this study, 10 years of Cluster data are analysed to determine the parameters of field-aligned chorus spectral characteristics as a function of latitude, in such a way that these parameters can be easily employed in numerical models to calculate the resulting diffusion rates. The measured statistics are in good agreement with our ray tracing simulations in a realistic model of Earth's inner magnetosphere, that explains the observed chorus spectral extent in terms of both wave growth/damping and cross- $L$ propagation. In the following sections we first present the field-aligned frequency distribution of chorus waves obtained from 10 years of measurements onboard Cluster spacecraft. Then we show how to model the spectral extent of chorus waves in the outer radiation belt using the ray tracing technique. Finally, we evaluate the impact of the frequency peak variation with latitude on electron diffusion rate, followed by some discussion and conclusions. In the Appendices the data set and analysis are described, as well as the numerical model employed in this study, in particular the energetic electron distributions used to reproduce wave growth/damping in the inner magnetosphere.

\section{Chorus wave spectral extent observed in the outer radiation belt}

In this section we first present the equatorial properties of chorus waves in the inner magnetosphere, observed by different spacecraft since first space missions, that are used to model the chorus "equatorial source region" in Sect. 3. Then we show the spectral extent of chorus waves as a function of latitude, obtained from statistics of 10 years of measurements by STAFF-SA instrument (Cornilleau-Wehrlin et al., 1997) onboard Cluster spacecraft, that are used to compare with numerical simulations and previous studies in Sect. 3 .

Chorus waves typically appear as short $(\sim 0.2 \mathrm{~s})$ coherent bursts of wave packets propagating in two distinct bands, scaling on the equatorial electron gyrofrequency $\Omega_{\mathrm{e} 0}$. The upper-band frequency range is generally observed to be $\omega \approx$ $0.5-0.7 \Omega_{\mathrm{e} 0}$ and the lower band frequency range is $\omega \approx 0.1-$ $0.45 \Omega_{\mathrm{e} 0}$ (see Burtis and Helliwell, 1969, 1976, for example). In this study, we show only results for the lower-band chorus waves because the full frequency range of the upper band cannot be entirely covered by Cluster STAFF-SA (see Agapitov et al., 2011). Moreover, upper-band chorus waves are substantially less intense than lower-band chorus waves (Meredith et al., 2001; Haque et al., 2010).

In Earth's magnetosphere, chorus waves are usually observed in the day, dawn and night sectors (Agapitov et al., 2011; Meredith et al., 2003a; Li et al., 2009) with a maximum in occurrence and wave intensity between 06:00 and 12:00 MLT (see e.g. Li et al., 2009; Agapitov et al., 2011). Additionally, the chorus source region is known to lie beyond the plasmapause and, as stated above, is generally located in 
the vicinity of the magnetic equator (see also LeDocq et al., 1998; Santolík et al., 2005a, b; Agapitov et al., 2010). Additionally, according to Agapitov et al. (2012), the equatorial $\theta_{0}$ distribution is not uniform but has a maximum at $\theta_{0} \approx 15^{\circ}$. Recent studies of chorus wave-normal distributions using extensive sets of observations from different spacecraft (see Agapitov et al., 2011; Li et al., 2011; Meredith et al., 2012) have also shown that the wave power distributions of intense chorus emissions have generally a non-zero mean value.

Recently, an extensive study (Li et al., 2013) presented for the first time the distribution of the azimuthal component of the wave-normal vector, for lower-band chorus waves at the equator as observed on THEMIS. Statistical measurements reveal that this distribution can be fitted by a Gaussian function in all MLT sectors, and particularly in the dawn sector with parameters $\phi_{m}=-3.4$ and $\delta \phi=50.84$, i.e. most waves are generated outward $\left(\phi=0^{\circ}\right)$. Chorus wave power distribution upon frequency is usually approximated at the equator by a Gaussian function with a peak value of $\omega_{m} \approx 0.34 \Omega_{\mathrm{e} 0}$ (and $\delta \omega \approx 0.15$ ), as has been observed on OGO1 and OGO3 (Burtis and Helliwell, 1969, 1976) spacecraft (see also Bunch et al., 2013).

However, in the off-equatorial magnetosphere the spectral extent of chorus waves is still not well known nor understood. As stated in the previous section, measurements of chorus spectral extent as a function of latitude have already been presented in Burtis and Helliwell (1969) and Bunch et al. (2013). However, statistics used in these studies are not substantial enough to cover the whole latitude $/ L$-shell range in which chorus waves are observed, as well as their whole lower-band spectrum.

Thus, in this section we process 10 years of magnetic and electric field wave power measured in a frequency range from $8 \mathrm{~Hz}$ to $4 \mathrm{kHz}$ by STAFF-SA instrument onboard Cluster spacecraft (see Appendix A and Agapitov et al., 2013, for details). These data, which cover a large range of magnetic latitudes $\left(0^{\circ} \leq \lambda \leq 45^{\circ}\right)$, are used to determine the lowerband chorus wave spectral power as a function of latitude. The obtained probability distribution function is presented in Fig. 1, where the global decrease of the frequency peak upon latitude is observed. In the vicinity of the equator $\left(\lambda \leq 3^{\circ}\right)$, the peak value of $\omega \approx 0.33 \Omega_{\mathrm{e} 0}$ confirms the results observed on OGO1 and OGO3 (Burtis and Helliwell, 1976) in the dayside although the peak value is slightly lower $\left(\sim 0.3 \Omega_{\mathrm{e} 0}\right)$ in the nightside. In both cases, a local minimum in chorus wave intensity is observed at the equator $\left(\lambda \leq 3^{\circ}\right)$, also in agreement with previous studies (Horne et al., 2005; Artemyev et al., 2012b). At low latitudes $\left(3^{\circ} \leq \lambda \leq 10^{\circ}\right)$ the peak value increases up to $\sim 0.4 \Omega_{\mathrm{e} 0}$ in the dayside, which indicates the presence of nonlinear wave-particle interaction (this point is discussed in Sect. 4), whereas in the nightside the peak value is steady in this latitude range. At mid-latitudes $\left(10^{\circ} \leq\right.$ $\lambda \leq 30^{\circ}$ ) the peak value in the dayside decreases almost linearly to $\sim 0.12 \Omega_{\mathrm{e} 0}$ at $\lambda \approx 25^{\circ}$ before a sharp inflection towards $\omega \approx 0.2 \Omega_{\mathrm{e} 0}$ is observed, consistent with results ob- tained by Bunch et al. (2013), whereas in the nightside the peak value dramatically drops to $\sim 0.15 \Omega_{\mathrm{e} 0}$ and stays constant up to the highest latitude $\left(40^{\circ}\right.$ here $)$. At high latitudes $\lambda>30^{\circ}$ in the dayside the peak frequency slowly decreases and its value reaches the value observed there in the nightside $\left(\sim 0.13 \Omega_{\mathrm{e} 0}\right)$, in agreement with previous results (Burtis and Helliwell, 1969; Ni et al., 2011). Moreover, the offequatorial chorus wave power is stronger on the dayside than on the nightside, which is consistent with previous statistics (see Meredith et al., 2012, and references therein).

The parameters obtained from Cluster statistics shown above, that can be used to calculate diffusion rates, are presented in Sect. 4, and in the following section we present the distribution obtained from numerical simulations in the dayside.

\section{Modelling the field-aligned spectral extent of chorus waves}

In this section we present how to model chorus spectral extent in the inner magnetosphere, using the numerical model described in the Appendix B (see also Breuillard et al., 2012a, b), in such a way that it can be compared with measurements carried out on Cluster (see Sect. 2) and POLAR (see Bunch et al., 2013) spacecraft. First, chorus wave power distribution is to be defined using realistic parameters from observations in the source region supposed to be at the equator (e.g. LeDocq et al., 1998; Santolík et al., 2005a; Agapitov et al., 2010). Then waves are numerically propagated through thermal (core and energetic) plasma by means of the ray tracing technique (Breuillard et al., 2012a, b), using the realistic model of the inner magnetosphere described above. Here we show that chorus frequency distribution as a function of latitude $\lambda$, observed by Cluster, can be quite well reproduced using numerical calculations of ray propagation from the chorus source region.

In order to reconstruct chorus frequency distribution statistics, we have calculated numerous ray trajectories in the inner magnetosphere (in terms of $L$-shell, latitude $\lambda$ and longitude $\varphi$ ). Rays are generated at the equator at $L=5$, where we launch a set of waves with frequencies in the range $0.1 \Omega_{\mathrm{e} 0} \leq \omega \leq 0.5 \Omega_{\mathrm{e} 0}$, spaced at intervals of $\Delta \omega=0.05 \Omega_{\mathrm{e} 0}$, to reproduce the lower-band chorus spectral extent. The 3-D obliqueness of chorus waves propagation is represented by the two angles $\theta$ and $\phi$, with $\theta$ being the latitudinal angle between $\boldsymbol{k}$-vector and magnetic field, and $\phi$ the azimuthal angle. For each $L_{0}$ and $\omega$, the range of initial angles $\phi_{0}$ between $\boldsymbol{k}$-vector and the background magnetic field $\boldsymbol{B}_{0}$ in the equatorial XY plane is $-180^{\circ} \leq \phi_{0} \leq+180^{\circ}$ with $\Delta \phi_{0}=10^{\circ}$, $\phi_{0}=0^{\circ}$ pointing away from the Earth. The range of initial wave-normal angles $\theta_{0}$ between $\boldsymbol{k}$-vector and $\boldsymbol{B}_{0}$ in the XZ plane is defined as $0 \leq \theta_{0} \leq 40^{\circ}$ since in the vicinity of the magnetic equator chorus waves are observed to be mostly 

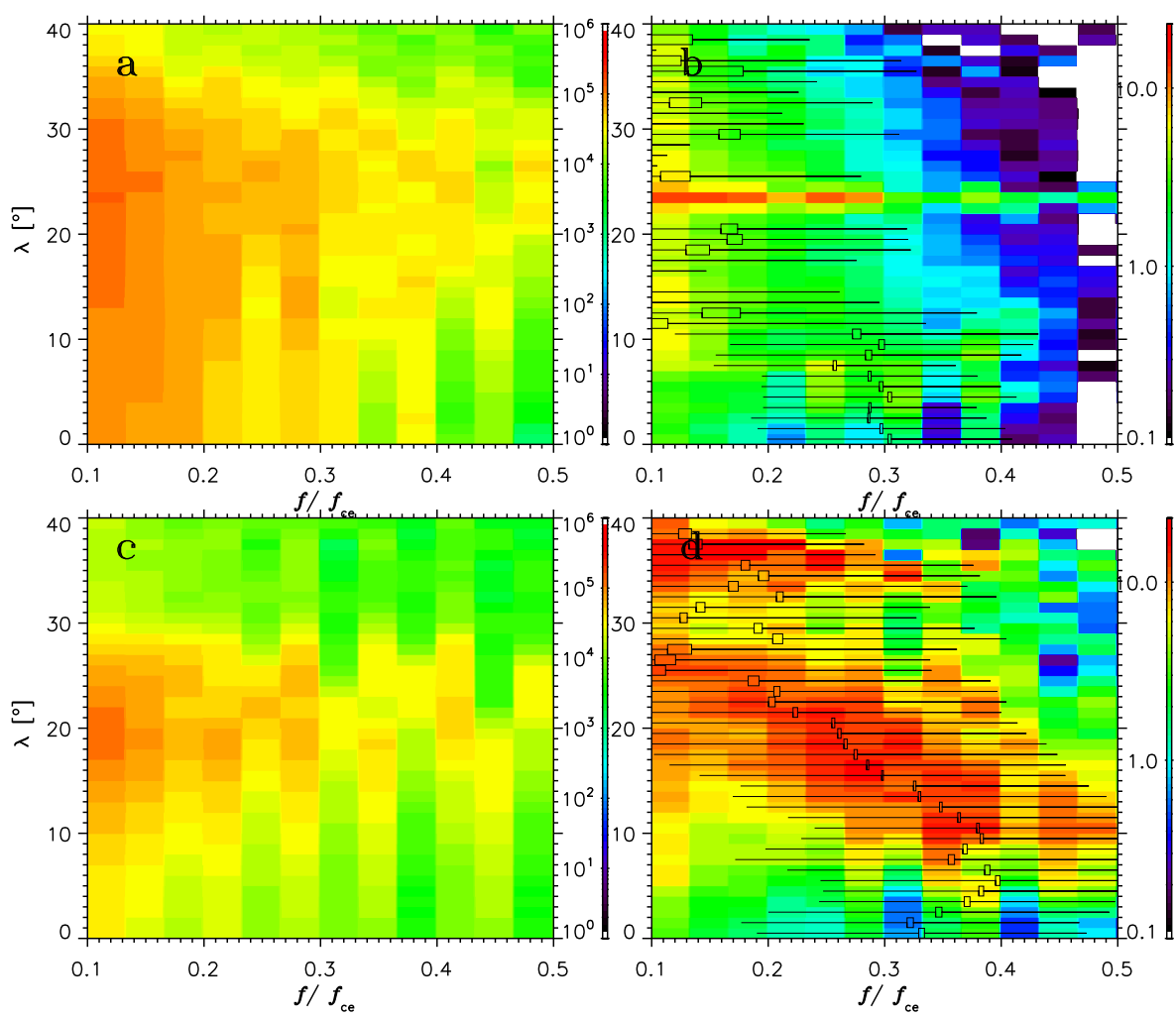

Figure 1. Number of measurements (left panels) and amplitude in pT (right panels) of chorus waves as a function of latitude obtained from 10 years of STAFF-SA instrument measurements onboard Cluster spacecraft on the nightside (top panels) and dayside (bottom panels) for $4<L<6$. The peak frequency (data is fitted by a Gaussian function) for each $1^{\circ}$ latitude range in right panels is indicated by a black rectangle.

quasi-parallel (see e.g. Agapitov et al., 2012). The interval step between each $\theta_{0}$ is $\Delta \theta_{0}=5^{\circ}$.

This numerical ray distribution is uniform in terms of $\omega$, $\theta_{0}$ and $\phi_{0}$ for the chorus wave frequency range, unlike the observed distributions described in Sect. 2. Our ray distribution thus needs to be intensity weighted at the injection points to properly model the chorus source region. This weighting is divided into three individual components representing the dependence on $\theta_{0}, \phi_{0}$ and $\omega$. Therefore, at the magnetic equator we use the typical chorus wave power distribution in frequency and azimuthal angle described in Sect. 2, and a weight function $h_{0}\left(\theta_{0}\right)$ for $\theta_{0}$ distribution which is simply a cross-section of the experimental distribution in Fig. 2e from Agapitov et al. (2012) at $\lambda=0^{\circ}$, for $0^{\circ}<\theta_{0}<90^{\circ}$.

Each ray is then propagated throughout the magnetospheric model described in Appendix B and recorded at different latitudes in a certain $L$-shell range, weighted in each point of its trajectory with an intensity given by its amplitude $A$ at this point. As a result, each ray trajectory is weighted with an intensity given by the following weight function:

$$
\begin{aligned}
g\left(\theta_{0}, \omega, \phi_{0}, A\right) & =h_{0}\left(\theta_{0}\right) \\
& \times \exp \left[-\frac{\left(\omega-0.34 \Omega_{\mathrm{e}, 0}\right)^{2}}{2\left(0.15 \Omega_{\mathrm{e}, 0}\right)^{2}}\right] \\
& \times \exp \left[-\frac{\left(\phi_{0}-(-3.4)\right)^{2}}{2(50.84)^{2}}\right] \cdot A,
\end{aligned}
$$

where the subscript 0 denotes the initial (equatorial) value.

The spectral extent of all rays is then reconstructed in Fig. 2 as a function of latitude, here in the same $L$-shell range $(4<L<6.5)$ as in Fig. 1, so that their peak frequency can be compared. Naturally, in the vicinity of the equator in Fig. 2, we do not reproduce the local minimum in wave intensity observed in Fig. 1 as our code does not reproduce such amplification observed at $3 \leq \lambda \leq 10^{\circ}$ in this figure. This can be explained by the fact that our model does not take into account (see Rönnmark, 1982; Breuillard et al., 2012b, for detailed explanation) the nonlinear mechanism thought to be responsible for chorus wave generation (see e.g. Dysthe, 1971; Omura et al., 1991, 2013; Trakhtengerts, 1999; Katoh and Omura, 2007, and references therein). This point is briefly discussed in the next section. Nevertheless, the global ten- 


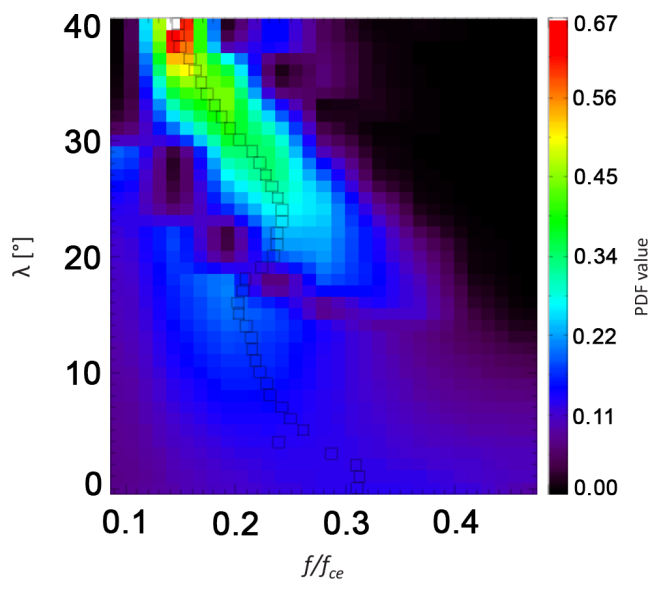

Figure 2. Power spectrum of chorus waves as a function of latitude obtained from our simulations for MLT $=09: 00,4<L<6$ and $K_{\mathrm{p}}=4$. The peak frequency (data is fitted by a Gaussian function) for each $1^{\circ}$ latitude range is indicated by a black square. Colourbar indicates the normalized PDF value.

dency of a decreasing peak frequency with increasing latitude, as well as the inflection at mid-latitudes, that is observed in Fig. 1 in the dayside (panel d) is reproduced in our simulations in Fig. 2.

In this figure, the peak frequency (squares) simulated in the dayside (MLT =09:00) decreases quite rapidly from $\omega \approx 0.33 \Omega_{\mathrm{e} 0}$ at the equator to $\omega \approx 0.2 \Omega_{\mathrm{e} 0}$ at $\lambda \approx 15^{\circ}$. Then, at mid-latitudes $\left(\lambda \approx 16-23^{\circ}\right)$ we observe an inflection of the peak frequency towards higher frequencies $\left(\sim 0.25 \Omega_{\mathrm{e} 0}\right)$ before it starts decreasing linearly down to $\sim 0.14 \Omega_{\mathrm{e} 0}$ at $\lambda=40^{\circ}$. It is interesting to note that, according to our simulations, the peak frequency value seems to be constant at very high latitudes $\left(\lambda>40^{\circ}\right)$. Thus, the peak frequency variations upon latitude obtained in our ray tracing simulations at MLT = 09:00 quite well reproduce the tendency observed in Cluster statistics in the dayside, i.e. the global decrease of the peak frequency with increasing latitude and the inflection at mid-latitudes. These results are also consistent with previous statistics obtained from different spacecraft, that are described in the following section. The explanation for such peak frequency variations with latitude in the frame of ray tracing technique is also presented in Sect. 4.

\section{Discussion}

In this paper we study the field-aligned spectral extent of chorus waves in the radiation belts by means of 10 years (20012010) of Cluster measurements and ray tracing simulations in a realistic model of Earth's inner magnetosphere. As suggested by Bunch et al. (2013), this study is crucial to sort out the competing effects of wave growth/damping and cross- $L$ propagation on chorus spectrum, and the parameters obtained have a significant impact on diffusion rate calculations in the outer radiation belt.

Here 10 years of statistics recorded onboard Cluster spacecraft in the dayside show a global decrease of the normalized frequency peak value with increasing latitude, from $\omega \approx 0.33 \Omega_{\mathrm{e} 0}$ near the equator to $\omega \approx 0.14 \Omega_{\mathrm{e} 0}$ at $\lambda=40^{\circ}$, in agreement with different previous studies (see e.g. Burtis and Helliwell, 1969; Ni et al., 2011). Moreover, we also observe an inflection of the peak value at middle latitude $\left(\lambda \approx 25^{\circ}\right)$, which is also consistent with results from a recent study using POLAR spacecraft data (Bunch et al., 2013). In our paper, these trends are confirmed by ray tracing simulations performed in the dayside (MLT $=09: 00$ ) of a realistic inner magnetosphere model, using observed properties of waves and particles during a typical chorus wave event.

These numerical simulations allow us to interpret the causes of such peak frequency variations observed on Cluster in terms of cross- $L$ propagation and wave growth/damping. First, near the magnetic equator $\left(\lambda \leq 10^{\circ}\right)$ the chorus frequency peak value $\left(\sim 0.33 \Omega_{\mathrm{e} 0}\right)$ results from the nonlinear mechanisms of chorus generation induced by "resonant" electrons $(\sim 10-100 \mathrm{keV})$ that are injected from the plasmasheet (see Omura et al., 2009, 2013; Summers et al., 2011, and references therein). Chorus waves are then amplified by this mechanism as they propagate away from equator, up to $\lambda \approx 10^{\circ}$ where "resonant" electrons are more scarce, resulting in a local wave intensity minimum at the equator (Horne et al., 2005; Artemyev et al., 2012b). In this latitude range on the dayside, the peak frequency is also observed to be shifted to $\sim 0.4 \Omega_{\mathrm{e} 0}$, that can be due to a decrease of the ratio $\omega_{\text {pe }} / \Omega_{e}$ with increasing latitude (Meredith et al., 2003b; Horne et al., 2005) which results in the increase of the carrier frequency of the nonlinear growth rate (see Fig. 1 in Omura et al., 2009). This effect is not reproduced in our simulations since nonlinear interactions are not taken into account in the code (see e.g. Rönnmark, 1982), and instead we observe a decrease of the peak frequency due to high-frequency wave damping, whereas low-frequency waves are linearly amplified (Kennel and Petschek, 1966).

At low/mid latitudes $\left(10^{\circ} \leq \lambda \leq 25^{\circ}\right)$, this rapid decrease is observed on Cluster since resonant electrons are scarcer there and high-frequency waves are thus preferably damped by "Landau" ( $\sim 0.1-1 \mathrm{keV})$ electrons (Kennel and Thorne, 1967). A sharp inflection of the peak value up to $\omega \geq 0.2 \Omega_{\mathrm{e} 0}$ at middle latitudes $\left(\lambda \approx 25^{\circ}\right)$ is then observed, in agreement with previous results obtained on POLAR (Bunch et al., 2013). This trend is also seen in the simulations, and can be explained by the fact that at mid-latitudes "high-frequency waves" $\left(\sim 0.3-0.5 \Omega_{\mathrm{e} 0}\right)$ are more damped than low-frequency waves $\left(\sim 0.1-0.2 \Omega_{\mathrm{e} 0}\right)$. However, the latter propagate mostly outward (towards higher $L$, see e.g. Breuillard et al., 2013) thus their normalized frequency is slightly increased (up to $\sim 0.2-0.25 \Omega_{\mathrm{e} 0}$ ). Finally, we observe a slow decrease of the peak frequency down to $\sim 0.13 \Omega_{\mathrm{e} 0}$ for higher latitudes $\left(\lambda \geq 30^{\circ}\right)$, due to the damping of the waves that remains (here 
Table 1. Coefficients of the quartic function used to fit the peak frequency as a function of latitude in the dayside and nightside.

\begin{tabular}{lrr}
\hline Coefficients $a_{i}$ & Dayside & Nightside \\
\hline$a_{0}$ & 0.29962728 & 0.29700697 \\
$a_{1}$ & 0.035828465 & 0.0075855753 \\
$a_{2}$ & -0.0041511747 & -0.0022720173 \\
$a_{3}$ & 0.00013766823 & 0.00010067242 \\
$a_{4}$ & $-1.4784885 \mathrm{e}-6$ & $-1.3031819 \mathrm{e}-6$ \\
\hline
\end{tabular}

also higher frequencies of such remaining waves, i.e. $\sim 0.2-$ $0.25 \Omega_{\mathrm{e} 0}$ are preferably damped out).

Cluster statistics in the nightside presented in this paper show a chorus peak frequency near the equator close $\left(\sim 0.3 \Omega_{\mathrm{e} 0}\right)$ to that observed in the dayside, indicating that the wave generation process (Omura et al., 2009, 2013; Summers et al., 2011) is similar. However, a dramatic drop in the peak frequency value $\left(\sim 0.13 \Omega_{\mathrm{e} 0}\right)$ for $\lambda>10^{\circ}$ is observed. This behaviour could be due to a simpler scenario: due to the strong electron fluxes (see e.g. Meredith et al., 2004; Bortnik et al., 2007; Li et al., 2010a) coming from the tail, the high frequencies $\left(\geq 0.2 \Omega_{\mathrm{e} 0}\right)$ of the chorus elements are heavily damped out (e.g. Bortnik et al., 2007; Chen et al., 2013) at $\lambda>10^{\circ}$, because the scarceness of "resonant" electrons in this region, unlike near the equator, cannot balance the strong damping of high-frequency waves by "Landau" electrons. In this off-equatorial region the peak frequency value is thus observed to be $\sim 0.13 \Omega_{\mathrm{e} 0}$ up to $\lambda=40^{\circ}$ (i.e. most of chorus frequencies have been damped), where the peak frequency in the dayside is similar. This frequency is very close to the typical frequency of hiss (i.e. whistler) waves, thus chorus waves recorded in this range and considered in this study can be mixed with hiss.

The behaviour of the chorus peak frequency as a function of latitude strongly affects the dynamics of the outer radiation belt. As a matter of fact, the distribution of mean wave frequency along field lines plays an important role for electron resonant scattering by waves. For each value of electron energy the most effective resonant interaction corresponds to a certain latitude domain. For example, $\mathrm{MeV}$ electrons with small equatorial pitch angles resonantly interact with whistler waves propagating parallel to the magnetic field at latitudes $\sim 30-40^{\circ}$ (Mourenas et al., 2012b; Artemyev et al., 2013a). Thus, the 3 times decrease of wave frequency between the equatorial plane and high latitudes can change the efficiency of scattering of small pitch angle electrons. To determine this effect we calculate pitch angle and energy diffusion rates, using the scheme of diffusion rate calculation described by Glauert and Horne (2005), for $1 \mathrm{MeV}$ electrons with constant mean wave frequency $\omega / \Omega_{\mathrm{e} 0}=0.35$ and with variation of $\omega$ with latitude presented in Fig. 1. To be able to implement the latter into the scheme of calculation, we fit the peak frequency variations as a function of latitude from
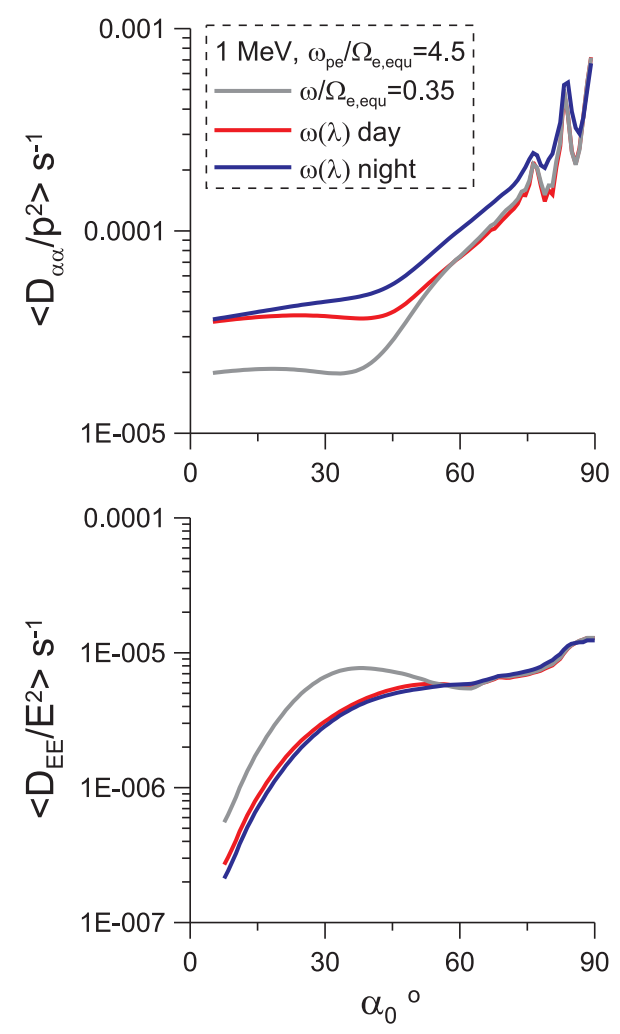

Figure 3. Pitch angle and energy diffusion coefficients as a function of electron equatorial pitch angle for $1 \mathrm{MeV}$ electrons interacting with parallel whistler waves (wave parameters are the same as in Glauert and Horne, 2005) at $L=5$. Grey colour shows results for "standard" constant wave frequency, while colour shows results for wave frequency varying with latitude (parameters from Table 1) as shown in Fig. 1 in the dayside (red) and nightside (blue).

Cluster statistics presented in Fig. 1 with a simple quartic (i.e. polynomial of degree 4) function for the dayside and nightside separately, and the coefficients of these quartic functions are shown in Table 1.

To follow the "standard" scheme (Glauert and Horne, 2005), we assume a constant wave magnetic field amplitude $B_{w}=100 \mathrm{pT}$, constant plasma frequency $\omega_{\mathrm{pe}} / \Omega_{\mathrm{e} 0}=4.5$ and dipole magnetic field at $L=5.0$. The comparison of the pitch angle and energy diffusion rates for dayside (red) and nightside (blue) with the standard constant value $\omega / \Omega_{\mathrm{e} 0}=0.35$ (grey) is shown in Fig. 3. One can see that the decrease of the wave peak frequency with the increase of latitude result in more effective electron scattering and less effective acceleration. The pitch angle diffusion coefficient increases by $\sim 40 \%$ and more than $50 \%$ for the dayside and the nightside, respectively, for relatively small equatorial pitch angles $\alpha_{\mathrm{eq}} \leq 45^{\circ}$ compared to the standard value. This significant effect should reduce the lifetime of these $\mathrm{MeV}$ electrons by $50 \%$. In the nightside the pitch angle diffusion is even increased for almost all pitch angles due to the drop of the peak frequency at $\lambda \approx 10^{\circ}$. In contrast to the increase of the pitch 
C1 PEACE (PITCH_SPIN_DEFluX)

2010-07-15T02:20:03.2572
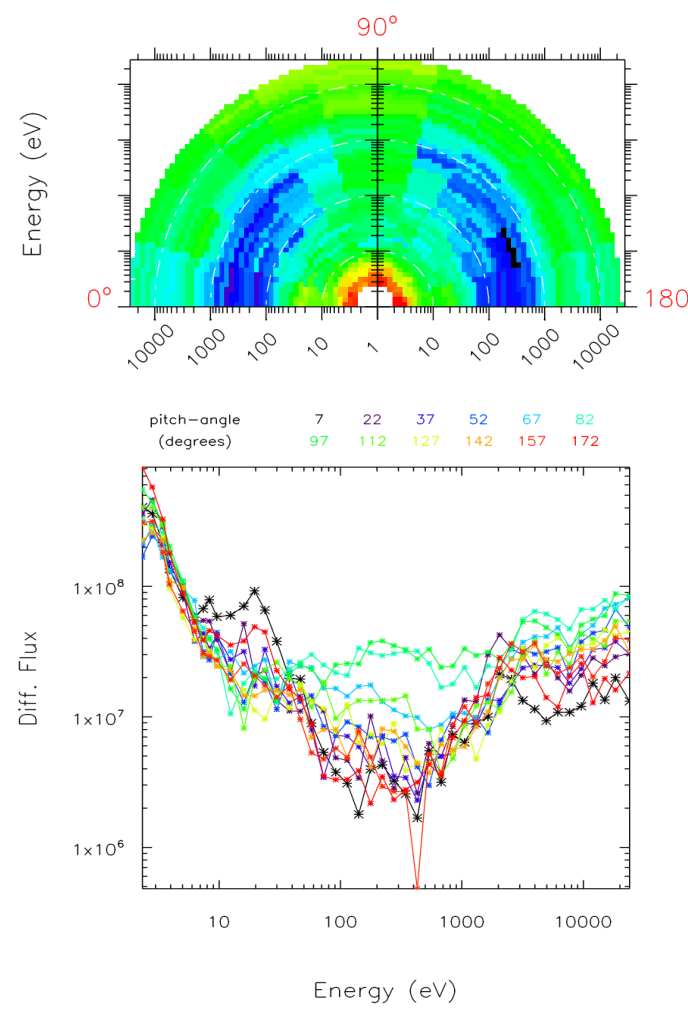

C1 RAPID (PAD_L3DD)

$2010-07-15$ T02:20:03.258Z
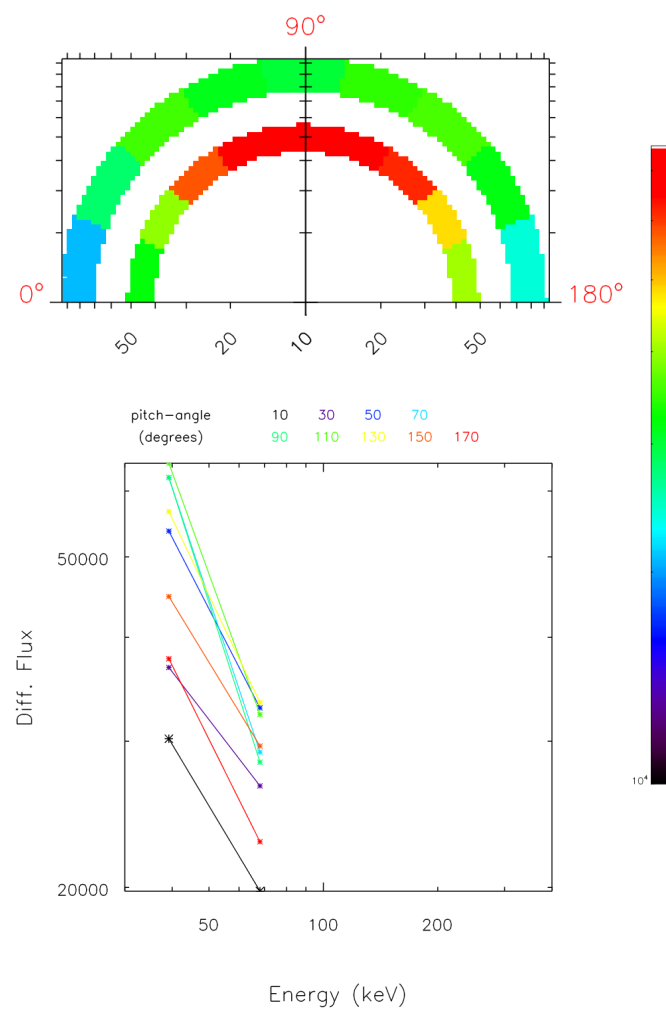

Figure 4. Equatorial distribution functions of a typical chorus event observed on 15 July 2010 onboard Cluster C1 at $L \approx 5$. Top panels present the electron wheel plots (distribution of pitch angle as a function of energy) measured by PEACE (upper-left panel) and RAPID (upper-right panel) instruments. Bottom panels show the electron differential fluxes (the different pitch angle ranges are colour-coded) as a function of energy observed during this event by the same instruments. These observed values are used to compute the electron distribution functions in our model.

angle diffusion coefficient, the energization rate decreases for relatively small pitch angles in the model with mean frequency varying with latitude for both dayside and nightside, compared to the constant frequency value.

The chorus wave spectral power presented in this study can thus be used to improve the accuracy of the diffusion coefficient calculations (see e.g. Summers, 2005; Shprits et al., 2006; Albert, 2007), which often assume that wave spectrum does not vary with latitude (see examples of exceptions in Summers and Ni, 2008; Mourenas et al., 2014; Ni et al., 2014). Additionally, our inner magnetospheric model could be useful to techniques aiming to construct the global distribution of chorus wave intensity (Ni et al., 2014), since simplified models of plasma density and magnetic field can lead to inaccuracies especially under active conditions (see e.g. Ni et al., 2014, and references therein).

\section{Conclusions}

The 10 years of Cluster statistics presented in this study are in agreement with previous measurements of chorus spectral extent (Burtis and Helliwell, 1969; Ni et al., 2011; Bunch et al., 2013), and well reproduced by the numerical simulations performed in this study. We show that the observed chorus wave spectral extent is resulting not only from cross- $L$ propagation, as suggested by Burtis and Helliwell (1969), but also from wave growth/damping as advanced by Bunch et al. (2013). Therewith, we demonstrate that the chorus spectral extent, in the range $0^{\circ} \leq \lambda \leq 40^{\circ}$, is mostly driven by wave growth/damping (decrease of the peak frequency) and outward propagation of low-frequency waves (inflection at midlatitudes), but the inward propagation of chorus waves only contributes very little at low latitudes - in contrast to the suggestion of Burtis and Helliwell (1969). The parameters obtained from Cluster statistics (see Table 1) are provided in such a way that they can be employed in any diffusion rate calculation to determine the effects of such chorus spec- 


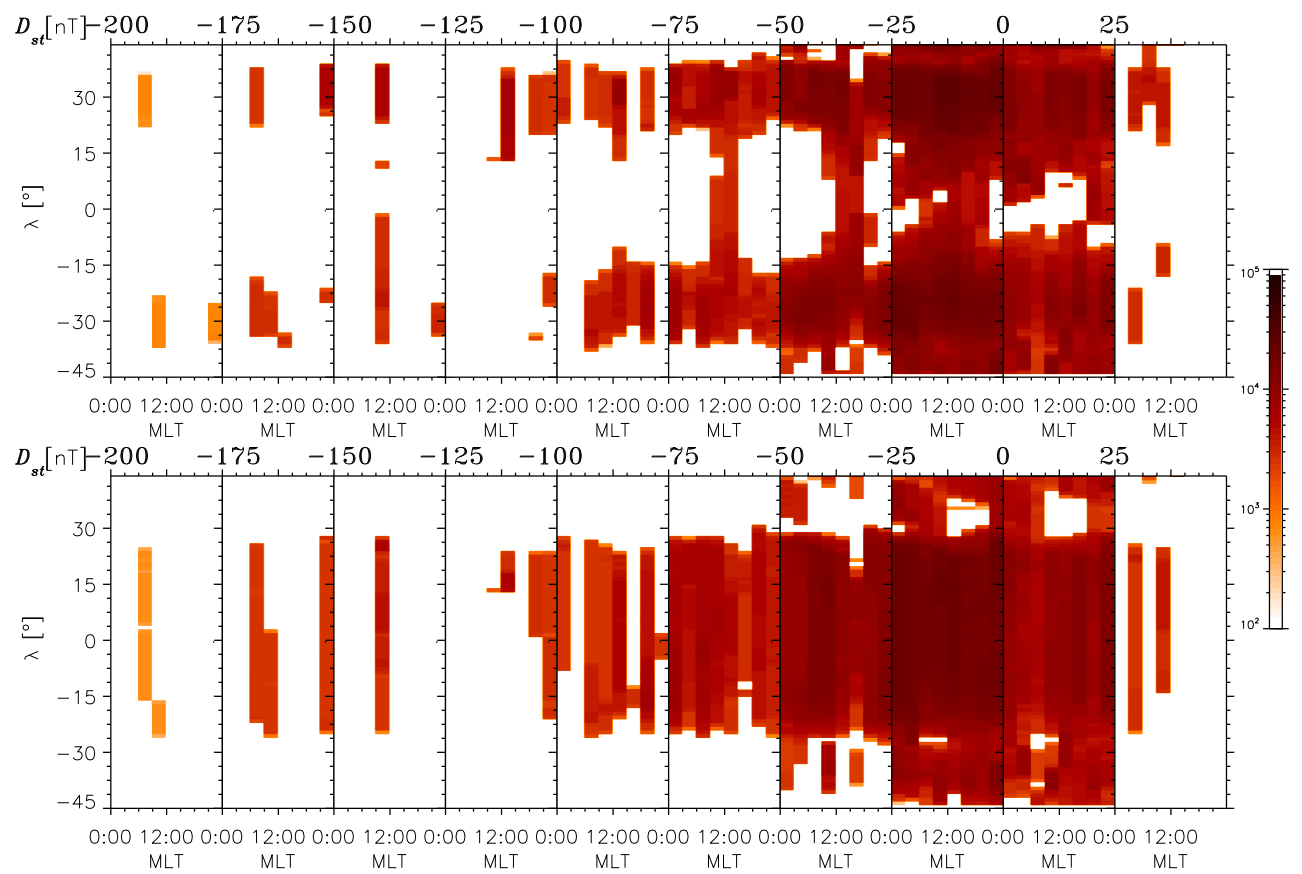

Figure 5. Data coverage (MLT as a function of $\lambda$ ) of the Cluster data set employed in this study, and described in detail in Agapitov et al. (2013), for different ranges of the Dst (Disturbance storm time) and $K_{\mathrm{p}}$ indices (top/bottom panel corresponds to $K_{\mathrm{p}}<3 / K_{\mathrm{p}}>3$, respectively.
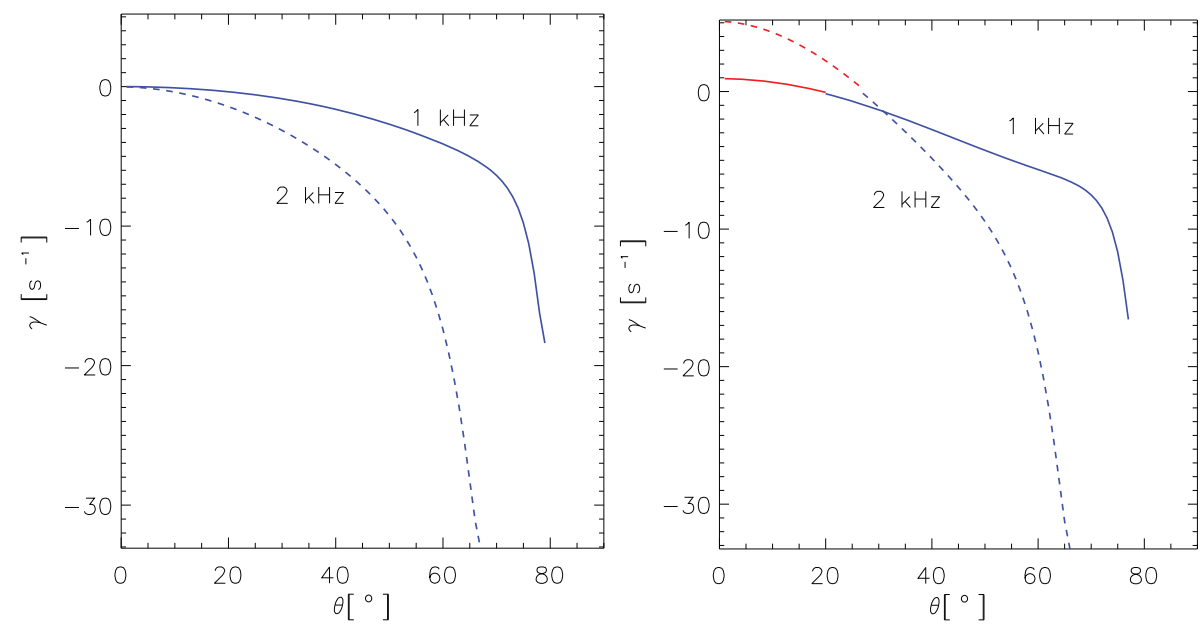

Figure 6. Damping (blue) and growth (red) rate $\gamma$ of 1 and $2 \mathrm{kHz}$ chorus waves at $L=5$ obtained by our simulations as a function of wave-normal angle $\theta$, using typical parameters described in Chen et al. (2013). On the left panel, only "Landau" ( $\sim 0.1-1 \mathrm{keV})$ electrons are included along with the core plasma, and on the right panel "resonant" ( $\sim 10-100 \mathrm{keV})$ electrons are also added.

tral extent more accurately. Its impact on $\mathrm{MeV}$ electrons is calculated in this study using the "standard" model (Glauert and Horne, 2005) of diffusion rates to compare with standard constant frequency. Results show, for both dayside and nightside, a decrease of the energization rate whereas pitch angle diffusion increases by $\sim 50 \%$ for relatively small pitch angles, which should reduce the lifetime of these electrons in the outer radiation belt by half.
Beside the electron resonant scattering by small-amplitude whistler waves, the nonlinear electron interaction with highamplitude waves (Bortnik et al., 2008; Artemyev et al., 2012c) also strongly affects the energetic electron dynamics. Two main resonances contributing to such nonlinear interaction are Landau and first cyclotron resonances. The wave frequency variation with latitude seems to be not very important for Landau resonance since phase velocity of oblique 
waves propagating with Gendrin angle does not depend on the wave frequency (Artemyev et al., 2013b). In contrast, for the first cyclotron resonance, observed and model wave frequency variation with latitude can be very important. The classical scenario assumes that electrons are trapped by highamplitude waves at high latitudes and are accelerated during the motion toward the equator. The total energy gain is proportional to change of wave frequency between trapping and escape latitudes (Demekhov et al., 2006; Tao et al., 2012). Thus, increase of the wave frequency between high latitudes and the equatorial plane should increase the efficiency of nonlinear acceleration. However, more detailed discussion of this topic is beyond the scope of the present study and should be subject to further investigation. 


\section{Appendix A: Data set and analysis}

In this study we partially use a large data set, described in detail in Agapitov et al. (2013), to compute the chorus wave spectral power as a function of latitude in Earth's outer radiation belt. The data set used here is composed of ELF/VLF waves observed in the range $|\lambda|<45^{\circ}$ and $4 \leq L \leq 6.5$ by Cluster spacecraft fleet between February 2001 and December 2010. Data are collected by the Spatio-Temporal Analysis of Field Fluctuations-Spectrum Analyzer (STAFF-SA) experiment (Cornilleau-Wehrlin et al., 2003), which provides the complete spectral matrix (real and imaginary parts) of the three magnetic field components measured by the STAFF search-coil magnetometer and the two components of wave electric field (Gustafsson et al., 2001). The survey includes STAFF-SA data from the Cluster 4 spacecraft (Samba) in order to avoid different statistical contributions due to different cross-spacecraft distances during the processing period. Spectral matrices and PSD data provided by Cluster Active Archive are used in SR2 (Spin Reference) frame. The spectral matrix was computed onboard for 27 frequency channels that were logarithmically spaced between $8.8 \mathrm{~Hz}$ and $3.56 \mathrm{kHz}$ (central frequencies), with the full range of $L$-shells and magnetic latitudes available for lower-band chorus. The considered wave frequency range $\left(0.1 f_{\text {ce }}<f<0.5 f_{\text {ce }}\right)$ in this study is indeed dominated by the lower-band chorus. Hiss waves can also be observed above $0.1 f_{\text {ce }}$, but their intensity rapidly falls above $1 \mathrm{kHz}$ (Meredith et al., 2004). The sensitivity of the STAFF searchcoil magnetometers is about $3.10^{-5} \mathrm{nT} / \mathrm{Hz}^{2}$ between $100 \mathrm{~Hz}$ and $4 \mathrm{kHz}$ (Cornilleau-Wehrlin et al., 2003). As stated in Agapitov et al. (2013), this data set contains a sufficient number of measurement points to perform a statistical study for the ranges of MLTs, $L$-shells, and $K_{\mathrm{p}}$ considered in this study (see Fig. 1 in Agapitov et al., 2013). We present also in Fig. 5 the data coverage of the employed data set as a function of the Dst index. Figure 5 shows that this data coverage is statistically significant even for high Dst values, and roughly indicates the number of storms included in the data points.

\section{Appendix B: The Earth's inner magnetosphere model}

To simulate the chorus wave power distribution upon frequency along a magnetic field line we use a 3-D ray-tracing code that includes a realistic model of the Earth's inner magnetosphere, described in detail in Breuillard et al. (2012b). The magnetic field and thermal plasma densities parameters - e.g. MLT $=09: 00$ and $K_{\mathrm{p}}=4$, see also Breuillard et al. (2012b) for details - are chosen to be typical of the period in which most chorus waves occur in the inner magnetosphere (Agapitov et al., 2011, 2013). Ray tracing (here the WHAMP solver) is sensitive to density variations in the chosen model. The trajectories of rays in the radiation belts are strongly affected by core plasma density gradients, and in particular the plasmapause (Wang et al., 2011), which are mostly driven by the $K_{\mathrm{p}}$ index value in this model (Gallagher et al., 2000). In fact, it has been shown (Zhou et al., 2011) that rays' trajectories (for $\lambda \leq 40^{\circ}$ ), launched at $L=6$ in this region, are not really modified in this model by the different $K_{\mathrm{p}}$ values, since rays do not approach plasmapause. However, in the range $4 \leq L \leq 6$ considered here, rays' trajectories could be affected during quiet times $\left(K_{\mathrm{p}}<3\right)$, since plasmapause can reach $L>4$ during those periods. This question, as well as a comparative study between magnetospheric core plasma models (Wang et al., 2011), should be addressed in a separate study. Nevertheless, a weaker chorus activity is observed for $K_{\mathrm{p}}<3$ (Agapitov et al., 2011, 2013), and making use of the "standard" parameters in this core plasma model, described in this study, appear to be suitable for studying chorus wave properties (see Breuillard et al., 2012a, b, 2014).

Density of suprathermal electrons has a small effect on rays' trajectories since, according to the measured statistics used in this study, the values of the two suprathermal electron populations $(\approx 10$ and $\approx 100 \mathrm{keV})$ are respectively 200 and 2000 times smaller than core $(0.5 \mathrm{eV})$ plasma density values at $L=5$ in the vicinity of the equator, for instance. While the cross- $L$ propagation weakly affects the wave power spectrum, as described in this study, and the effect of core plasma is negligible on the rays' amplitudes (see e.g. Breuillard et al., 2014), the damping/amplification by suprathermal electrons plays a primary role in defining chorus wave distributions in the radiation belts (Bortnik et al., 2006, 2007; Chen et al., 2013). In order to accurately reproduce the variations of the waves' amplitude (i.e. damping/growth) during their propagation, in this study we include two populations of energetic electrons into the code, in addition to the four background species. The energies defining each electron population are described in terms of a Maxwellian function (Rönnmark, 1982), whose maximum value is chosen to be typically resonant with chorus waves in the inner magnetosphere, i.e. to be responsible for chorus wave damping/growth in the radiation belts. In this region, the socalled "Landau electrons" (roughly from $100 \mathrm{eV}$ to $1 \mathrm{keV}$ ) are thought to be mainly responsible for the Landau damping of chorus waves (see e.g. Kennel and Thorne, 1967), whereas "resonant electrons" $(\sim 10-100 \mathrm{keV})$ are considered to imply linear (as well as nonlinear) chorus wave growth (see e.g. Kennel and Petschek, 1966). The values of omnidirectional fluxes for each population used in this study are based on observed properties of a typical chorus event recorded on 15 July 2010 at 02:20:00 UT onboard Cluster C1 (see bottom panels of Fig. 4), which was located in the vicinity of the magnetic equator $\left(\lambda=-0.4^{\circ}\right)$ at $L \approx 5$ in the morning side $(M L T=07: 30)$ at that time. The values of anisotropy for each population are also based on measurements from PEACE (Johnstone et al., 1997) and RAPID (Wilken et al., 1997) instruments onboard C1 during this event (see top panels of Fig. 4). The anisotropy observed is about 5 for "Landau electrons" and 2.5 for "resonant electrons", which is con- 
sistent with measurements of such electron populations performed on THEMIS during typical chorus events (see e.g. Li et al., 2010b; Cully et al., 2011).

The density of these two suprathermal electron populations in the inner magnetosphere has then to be defined in order to include them into our model. The density value for each population is taken from statistics at the equator (depending on $K_{\mathrm{p}}$ value) measured by MEPED and TED sensors onboard NOAA spacecraft (D. Boscher and S. Bourdarie, private communication, 2011), and is computed along the closed magnetic field lines (in our case for $4 \leq L \leq 7$ ) taking as a basis a theoretical model of electron density along the dipolar field lines in the radiation belts. A 2-D density grid has thus been generated and implemented into our global model of the inner magnetosphere, in addition to the different core plasma species. Making use of the density grids of all particle populations, and implementing their parameters described in the previous paragraph into the WHAMP solver, it is possible to obtain the imaginary part $\Im \omega$ of the frequency (i.e. the growth/damping rate $\gamma$ ) from which one can deduce the amplitude $A$ of the wave (see Breuillard et al., 2012b).
The growth/damping rate $\gamma$, obtained using our model with typical chorus wave parameters in the radiation belts, i.e. $L=5, \mathrm{MLT}=09: 00, f=1,2 \mathrm{kHz}$ and $K_{\mathrm{p}}=4$ (see e.g. Breuillard et al., 2012b; Chen et al., 2013, and references therein), is presented in Fig. 6 as a function of the wavenormal angle $\theta$. In Fig. 6, the damping rates (negative $\gamma$, in blue) are consistent (in particular when adding resonant electrons in Fig. 1b) with the one obtained in Fig. 2 by Chen et al. (2013), where similar parameters for the "Landau" electrons are used but in a different model. Note that damping rates obtained using our model at $L=6$ (not shown) are also consistent with results obtained by Chen et al. (2013) in Fig. 2. However, by adding into our model the "resonant" electron population one can see in Fig. $6 \mathrm{~b}$ that we obtain also a growth rate (positive $\gamma$, in red) for quasi-parallel waves $\left(\theta \leq 25^{\circ}\right)$, which means that quasi-parallel waves are amplified by the resonant electrons, as predicted by the quasi-linear theory (see e.g. Kennel and Petschek, 1966; Ginzburg and Rukhadze, 1975). These results are in good agreement with previous results that have shown that whistler wave damping (Bortnik et al., 2006; Chen et al., 2013) and amplification (Chen et al., 2012; Watt et al., 2012) can be studied using quasi-linear theory and ray tracing. Thus, our model makes it possible to study the combined effects of damping and amplification on chorus wave propagation properties for the first time in a realistic model of the inner magnetosphere. 
Acknowledgements. The authors would like to thank Mikhail Rashev for the help in RAPID data calibrations. The work by O. Agapitov was performed under JHU/APL Contract No. 922613 (RBSP-EFW) and NASA NNX09AE41G-1/14 Contract. The work of A. Artemyev was partially supported by the grant MK1781.2014.2.

The article processing charges for this open-access publication were covered by the Max Planck Society.

The topical editor C. Owen thanks the two anonymous referees for help in evaluating this paper.

\section{References}

Agapitov, O., Krasnoselskikh, V., Zaliznyak, Yu., Angelopoulos, V., Le Contel, O., and Rolland, G.: Chorus source region localization in the Earth's outer magnetosphere using THEMIS measurements, Ann. Geophys., 28, 1377-1386, doi:10.5194/angeo28-1377-2010, 2010.

Agapitov, O., Krasnoselskikh, V., Khotyaintsev, Y. V., and Rolland, G.: A statistical study of the propagation characteristics of whistler waves observed by Cluster, Geophys. Res. Lett., 38, L20103, doi:10.1029/2011GL049597, 2011.

Agapitov, O., Krasnoselskikh, V., Khotyaintsev, Y. V., and Rolland, G.: Correction to "A statistical study of the propagation characteristics of whistler waves observed by Cluster", Geophys. Res. Lett., 39, L24102, doi:10.1029/2012GL054320, 2012.

Agapitov, O., Artemyev, A., Krasnoselskikh, V., Khotyaintsev, Y. V., Mourenas, D., Breuillard, H., Balikhin, M., and Rolland, G.: Statistics of whistler-mode waves in the outer radiation belt: Cluster STAFF-SA measurements, J. Geophys. Res.-Space, 118, 3407-3420, doi:10.1002/jgra.50312, 2013.

Albert, J. M.: Simple approximations of quasi-linear diffusion coefficients, J. Geophys. Res.-Space, 112, A12202, doi:10.1029/2007JA012551, 2007.

Artemyev, A., Agapitov, O., Breuillard, H., Krasnoselskikh, V., and Rolland, G.: Electron pitch-angle diffusion in radiation belts: The effects of whistler wave oblique propagation, Geophys. Res. Lett., 39, L08105, doi:10.1029/2012GL051393, 2012a.

Artemyev, A., Agapitov, O., Krasnoselskikh, V., Breuillard, H., and Rolland, G.: Statistical model of electron pitch angle diffusion in the outer radiation belt, J. Geophys. Res.-Space, 117, A08219, doi:10.1029/2012JA017826, 2012b.

Artemyev, A., Krasnoselskikh, V., Agapitov, O., Mourenas, D., and Rolland, G.: Non-diffusive resonant acceleration of electrons in the radiation belts., Phys. Plasmas, 19, 122901, doi:10.1063/1.4769726, 2012c.

Artemyev, A. V., Mourenas, D., Agapitov, O. V., and Krasnoselskikh, V. V.: Parametric validations of analytical lifetime estimates for radiation belt electron diffusion by whistler waves, Ann. Geophys., 31, 599-624, doi:10.5194/angeo-31-599-2013, 2013a.

Artemyev, A. V., Vasiliev, A. A., Mourenas, D., Agapitov, O., and Krasnoselskikh, V.: Nonlinear electron acceleration by oblique whistler waves: Landau resonance vs. cyclotron resonance., Phys. Plasmas, 20, 122901, doi:10.1063/1.4836595, 2013b.
Baker, D. N.: How to Cope with Space Weather, Science, 297, 1486-1487, doi:10.1126/science.1074956, 2002.

Bortnik, J. and Thorne, R. M.: The dual role of ELF/VLF chorus waves in the acceleration and precipitation of radiation belt electrons, J. Atmos. Sol.-Terr. Phy., 69, 378-386, doi:10.1016/j.jastp.2006.05.030, 2007.

Bortnik, J., Inan, U. S., and Bell, T. F.: Landau damping and resultant unidirectional propagation of chorus waves, Geophys. Res. Lett., 33, L03102, doi:10.1029/2005GL024553, 2006.

Bortnik, J., Thorne, R. M., and Meredith, N. P.: Modeling the propagation characteristics of chorus using CRRES suprathermal electron fluxes, J. Geophys. Res. Space, 112, A08204, doi:10.1029/2006JA012237, 2007.

Bortnik, J., Thorne, R. M., and Inan, U. S.: Nonlinear interaction of energetic electrons with large amplitude chorus, Geophys. Res. Lett., 35, L21102, doi:10.1029/2008GL035500, 2008.

Bourdarie, S., Boscher, D., Beutier, T., Sauvaud, J.-A., and Blanc, M.: Magnetic storm modeling in the Earth's electron belt by the Salammbô code, J. Geophys. Res., 101, 27171-27176, doi:10.1029/96JA02284, 1996.

Breuillard, H., Mendzhul, D., and Agapitov, O.: Effects of equatorial chorus wave normal azimuthal distribution on wave propagation, Adv. Astron. Space Phys., 2, 167-172, 2012a.

Breuillard, H., Zaliznyak, Y., Krasnoselskikh, V., Agapitov, O., Artemyev, A., and Rolland, G.: Chorus wave-normal statistics in the Earth's radiation belts from ray tracing technique, Ann. Geophys., 30, 1223-1233, doi:10.5194/angeo-30-1223-2012, 2012b.

Breuillard, H., Zaliznyak, Y., Agapitov, O., Artemyev, A., Krasnoselskikh, V., and Rolland, G.: Spatial spreading of magnetospherically reflected chorus elements in the inner magnetosphere, Ann. Geophys., 31, 1429-1435, doi:10.5194/angeo-311429-2013, 2013.

Breuillard, H., Agapitov, O., Artemyev, A., Krasnoselskikh, V., Le Contel, O., Cully, C. M., Angelopoulos, V., Zaliznyak, Y., and Rolland, G.: On the origin of falling-tone chorus elements in Earth's inner magnetosphere, Ann. Geophys., 32, 1477-1485, doi:10.5194/angeo-32-1477-2014, 2014.

Bunch, N. L., Spasojevic, M., and Shprits, Y. Y.: Off-equatorial chorus occurrence and wave amplitude distributions as observed by the Polar Plasma Wave Instrument, J. Geophys. Res.-Space, 117, A04205, doi:10.1029/2011JA017228, 2012.

Bunch, N. L., Spasojevic, M., Shprits, Y. Y., Gu, X., and Foust, F.: The spectral extent of chorus in the off-equatorial magnetosphere, J. Geophys. Res.-Space, 118, 1700-1705, doi:10.1029/2012JA018182, 2013.

Burtis, W. J. and Helliwell, R. A.: Banded chorus - A new type of VLF radiation observed in the magnetosphere by OGO 1 and OGO 3., J. Geophys. Res., 74, 3002-3010, doi:10.1029/JA074i011p03002, 1969.

Burtis, W. J. and Helliwell, R. A.: Magnetospheric chorus - Occurrence patterns and normalized frequency, Plan. Sp. Sci., 24, 1007-1024, doi:10.1016/0032-0633(76)90119-7, 1976.

Chen, L., Li, W., Bortnik, J., and Thorne, R. M.: Amplification of whistler-mode hiss inside the plasmasphere, Geophys. Res. Lett., 39, L08111, doi:10.1029/2012GL051488, 2012.

Chen L., Thorne, R. M., Li, W., and Bortnik, J.: Modeling the wave normal distribution of chorus waves, J. Geophys. Res.-Space, 118, 1074-1088, doi:10.1029/2012JA018343, 2013. 
Chen, Y., Reeves, G. D., and Friedel, R. H. W.: The energization of relativistic electrons in the outer Van Allen radiation belt, Nature Physics, 3, 614-617, doi:10.1038/nphys655, 2007.

Cornilleau-Wehrlin, N., Chauveau, P., Louis, S., Meyer, A., Nappa, J. M., Perraut, S., Rezeau, L., Robert, P., Roux, A., de Villedary, C., de Conchy, Y., Friel, L., Harvey, C. C., Hubert, D., Lacombe, C., Manning, R., Wouters, F., Lefeuvre, F., Parrot, M., Pincon, J. L., Poirier, B., Kofman, W., and Louarn, P.: The Cluster SpatioTemporal Analysis of Field Fluctuations (STAFF) Experiment, Space Sci. Rev., 79, 107-136, doi:10.1023/A:1004979209565, 1997.

Cornilleau-Wehrlin, N., Chanteur, G., Perraut, S., Rezeau, L., Robert, P., Roux, A., de Villedary, C., Canu, P., Maksimovic, M., de Conchy, Y., Hubert, D., Lacombe, C., Lefeuvre, F., Parrot, M., Pinçon, J. L., Décréau, P. M. E., Harvey, C. C., Louarn, Ph., Santolik, O., Alleyne, H. St. C., Roth, M., Chust, T., Le Contel, O., and STAFF team: First results obtained by the Cluster STAFF experiment, Ann. Geophys., 21, 437-456, doi:10.5194/angeo-21437-2003, 2003.

Cully, C. M., Angelopoulos, V., Auster, U., Bonnell, J., and Le Contel, O.: Observational evidence of the generation mechanism for rising-tone chorus, Geophys. Res. Lett., 38, L01106, doi:10.1029/2010GL045793, 2011.

Daglis, I., Baker, D., Kappenman, J., Panasyuk, M., and Daly, E.: Effects of space weather on technology infrastructure, Space Weather, 2, S02004, doi:10.1029/2003SW000044, 2004.

Demekhov, A. G., Trakhtengerts, V. Y., Rycroft, M. J., and Nunn, D.: Electron acceleration in the magnetosphere by whistler-mode waves of varying frequency, Geomagn. Aeronomy+, 46, 711716, doi:10.1134/S0016793206060053, 2006.

Dysthe, K. B.: Some studies of triggered whistler emissions, J. Geophys. Res., 76, 6915, doi:10.1029/JA076i028p06915, 1971.

Fok, M.-C., Horne, R. B., Meredith, N. P., and Glauert, S. B.: Radiation Belt Environment model: Application to space weather nowcasting, J. Geophys. Res., 113, A03S08, doi:10.1029/2007JA012558, 2008.

Gallagher, D. L., Craven, P. D., and Comfort, R. H.: Global core plasma model, J. Geophys. Res., 105, 18819-18834, doi:10.1029/1999JA000241, 2000.

Ginzburg, V. L. and Rukhadze, A. A.: Waves in magnetoactive plasma, Nauka, Moscow, 2nd revised edn., 1975.

Glauert, S. B. and Horne, R. B.: Calculation of pitch angle and energy diffusion coefficients with the PADIE code, J. Geophys. Res., 110, A04206, doi:10.1029/2004JA010851, 2005.

Gustafsson, G., André, M., Carozzi, T., Eriksson, A. I., Fälthammar, C.-G., Grard, R., Holmgren, G., Holtet, J. A., Ivchenko, N., Karlsson, T., Khotyaintsev, Y., Klimov, S., Laakso, H., Lindqvist, P.-A., Lybekk, B., Marklund, G., Mozer, F., Mursula, K., Pedersen, A., Popielawska, B., Savin, S., Stasiewicz, K., Tanskanen, P., Vaivads, A., and Wahlund, J.-E.: First results of electric field and density observations by Cluster EFW based on initial months of operation, Ann. Geophys., 19, 1219-1240, doi:10.5194/angeo19-1219-2001, 2001.

Haque, N., Spasojevic, M., Santolík, O., and Inan, U. S.: Wave normal angles of magnetospheric chorus emissions observed on the Polar spacecraft, J. Geophys. Res., 115, A00F07, doi:10.1029/2009JA014717, 2010.
Horne, R. B.: The contribution of wave-particle interactions to electron loss and acceleration in the Earths radiation belts during geomagnetic storms, Review of Radio Science, 33, 801, 2002.

Horne, R. B., Thorne, R. M., Glauert, S. A., Albert, J. M., Meredith, N. P., and Anderson, R. R.: Timescale for radiation belt electron acceleration by whistler mode chorus waves, J. Geophys. Res., 110, A03225, doi:10.1029/2004JA010811, 2005.

Iucci, N., Levitin, A. E., Belov, A. V., Eroshenko, E. A., Ptitsyna, N. G., Villoresi, G., Chizhenkov, G. V., Dorman, L. I., Gromova, L. I., Parisi, M., Tyasto, M. I., and Yanke, V. G.: Space weather conditions and spacecraft anomalies in different orbits, Space Weather, 3, S01001, doi:10.1029/2003SW000056, 2005.

Johnstone, A. D., Alsop, C., Burge, S., Carter, P. J., Coates, A. J., Coker, A. J., Fazakerley, A. N., Grande, M., Gowen, R. A., Gurgiolo, C., Hancock, B. K., Narheim, B., Preece, A., Sheather, P. H., Winningham, J. D., and Woodliffe, R. D.: Peace: a Plasma Electron and Current Experiment, Space Sci. Rev., 79, 351-398, doi:10.1023/A:1004938001388, 1997.

Katoh, Y. and Omura, Y.: Computer simulation of chorus wave generation in the Earth's inner magnetosphere, Geophys. Res. Lett., 34, L03102, doi:10.1029/2006GL028594, 2007.

Kennel, C. F. and Petschek, H. E.: Limit on Stably Trapped Particle Fluxes, J. Geophys. Res., 71, 1, 1966.

Kennel, C. F. and Thorne, R. M.: Unstable growth of unducted whistlers propagating at an angle to the geomagnetic field, J. Geophys. Res., 72, 871-878, doi:10.1029/JZ072i003p00871, 1967.

Lanzerotti, L. J.: Space Weather and Natural Hazards, Space Weather, 10, S05008, doi:10.1029/2012SW000810, 2012.

LeDocq, M. J., Gurnett, D. A., and Hospodarsky, G. B.: Chorus Source Locations from VLF Poynting Flux Measurements with the Polar Spacecraft, Geophys. Res. Lett., 25, 4063, doi:10.1029/1998GL900071, 1998.

Li, W., Thorne, R. M., Angelopoulos, V., Bortnik, J., Cully, C. M., Ni, B., LeContel, O., Roux, A., Auster, U., and Magnes, W.: Global distribution of whistler-mode chorus waves observed on the THEMIS spacecraft, Geophys. Res. Lett., 36, L09104, doi:10.1029/2009GL037595, 2009.

Li, W., Thorne, R. M., Bortnik, J., Nishimura, Y., Angelopoulos, V., Chen, L., McFadden, J. P., and Bonnell, J. W.: Global distributions of suprathermal electrons observed on THEMIS and potential mechanisms for access into the plasmasphere, J. Geophys. Res.-Space, 115, A00J10, doi:10.1029/2010JA015687, 2010a.

Li, W., Thorne, R. M., Nishimura, Y., Bortnik, J., Angelopoulos, V., McFadden, J. P., Larson, D. E., Bonnell, J. W., Le Contel, O., Roux, A., and Auster, U.: THEMIS analysis of observed equatorial electron distributions responsible for the chorus excitation, J. Geophys. Res.-Space, 115, A00F11, doi:10.1029/2009JA014845, 2010b.

Li, W., Bortnik, J., Thorne, R. M., and Angelopoulos, V.: Global distribution of wave amplitudes and wave normal angles of chorus waves using THEMIS wave observations, J. Geophys. Res., 116, A12205, doi:10.1029/2011JA017035, 2011.

Li, W., Bortnik, J., Thorne, R. M., Cully, C. M., Chen, L., Angelopoulos, V., Nishimura, Y., Tao, J. B., Bonnell, J. W., and LeContel, O.: Characteristics of the Poynting flux and wave normal vectors of whistler-mode waves observed on THEMIS, J. Geophys. Res.-Space, 118, 1461-1471, doi:10.1002/jgra.50176, 2013. 
Li, X., Temerin, M., Baker, D. N., Reeves, G. D., and Larson, D.: Quantitative prediction of radiation belt electrons at geostationary orbit based on solar wind measurements, Geophys. Res. Lett., 28, 1887-1890, doi:10.1029/2000GL012681, 2001.

Lyons, L. R.: General relations for resonant particle diffusion in pitch angle and energy, J. Plasma Phys., 12, 45, doi:10.1017/S0022377800024910, 1974.

Lyons, L. R. and Williams, D. J.: Quantitative aspects of magnetospheric physics., D. Reidel Publishing Company, Springer Netherlands, 133-228, 1984.

Meredith, N. P., Horne, R. B., and Anderson, R. R.: Substorm dependence of chorus amplitudes: Implications for the acceleration of electrons to relativistic energies, J. Geophys. Res., 106, 13165-13178, doi:10.1029/2000JA900156, 2001.

Meredith, N. P., Cain, M., Horne, R. B., Thorne, R. M., Summers, D., and Anderson, R. R.: Evidence for chorus-driven electron acceleration to relativistic energies from a survey of geomagnetically disturbed periods, J. Geophys. Res., 108, 1248, doi:10.1029/2002JA009764, 2003a.

Meredith, N. P., Horne, R. B., Thorne, R. M., and Anderson, R. R.: Favored regions for chorus-driven electron acceleration to relativistic energies in the Earth's outer radiation belt, Geophys. Res. Lett., 30, 1871, doi:10.1029/2003GL017698, 2003 b.

Meredith, N. P., Horne, R. B., Thorne, R. M., Summers, D., and Anderson, R. R.: Substorm dependence of plasmaspheric hiss, J. Geophys. Res.-Space, 109, A06209, doi:10.1029/2004JA010387, 2004.

Meredith, N. P., Horne, R. B., Sicard-Piet, A., Boscher, D., Yearby, K. H., Li, W., and Thorne, R. M.: Global model of lower band and upper band chorus from multiple satellite observations, J. Geophys. Res.-Space, 117, A10225, doi:10.1029/2012JA017978, 2012.

Mourenas, D., Artemyev, A., Agapitov, O., and Krasnoselskikh, V.: Acceleration of radiation belts electrons by oblique chorus waves, J. Geophys. Res.-Space, 117, A10212, doi:10.1029/2012JA018041, 2012a.

Mourenas, D., Artemyev, A. V., Ripoll, J.-F., Agapitov, O. V., and Krasnoselskikh, V. V.: Timescales for electron quasi-linear diffusion by parallel and oblique lower-band Chorus waves., J. Geophys. Res., 117, A06234, doi:10.1029/2012JA017717, 2012 b.

Mourenas, D., Artemyev, A. V., Agapitov, O. V., and Krasnoselskikh, V.: Consequences of geomagnetic activity on energization and loss of radiation belt electrons by oblique chorus waves, J. Geophys. Res.-Space, 119, 2775-2796, doi:10.1002/2013JA019674, 2014.

Ni, B., Thorne, R. M., Meredith, N. P., Shprits, Y. Y., and Horne, R. B.: Diffuse auroral scattering by whistler mode chorus waves: Dependence on wave normal angle distribution, J. Geophys. Res.-Space, 116, A10207, doi:10.1029/2011JA016517, 2011.

Ni, B., Li, W., Thorne, R. M., Bortnik, J., Green, J. C., Kletzing, C. A., Kurth, W. S., Hospodarsky, G. B., and Soria-Santacruz Pich, M.: A novel technique to construct the global distribution of whistler mode chorus wave intensity using low-altitude POES electron data, J. Geophys. Res.-Space, 119, 5685-5699, doi:10.1002/2014JA019935, 2014.

Omura, Y., Matsumoto, H., Nunn, D., and Rycroft, M. J.: A review of observational, theoretical and numerical studies of VLF triggered emissions, J. Atmos. Terr. Phys., 53, 351-368, 1991.
Omura, Y., Hikishima, M., Katoh, Y., Summers, D., and Yagitani, S.: Nonlinear mechanisms of lower-band and upper-band VLF chorus emissions in the magnetosphere, J. Geophys. Res.-Space, 114, A07217, doi:10.1029/2009JA014206, 2009.

Omura, Y., Nunn, D., and Summers, D.: Generation Processes of Whistler Mode Chorus Emissions: Current Status of Nonlinear Wave Growth Theory, 243-254, American Geophysical Union, doi:10.1029/2012GM001347, 2013.

Rönnmark, K.: Computation of the dielectric tensor of a Maxwellian plasma, 1-60, KGI Report N. 179, Kiruna, Sweden, 1982.

Santolík, O., Gurnett, D. A., Pickett, J. S., Parrot, M., and Cornilleau-Wehrlin, N.: Central position of the source region of storm-time chorus, Plan. Sp. Sci., 53, 299-305, doi:10.1016/j.pss.2004.09.056, 2005a.

Santolík, O., Macúšová, E., Yearby, K. H., Cornilleau-Wehrlin, N., and Alleyne, H. StC. K.: Radial variation of whistler-mode chorus: first results from the STAFF/DWP instrument on board the Double Star TC-1 spacecraft, Ann. Geophys., 23, 2937-2942, doi:10.5194/angeo-23-2937-2005, 2005b.

Santolík, O., Gurnett, D. A., Pickett, J. S., Grimald, S., Décreau, P. M. E., Parrot, M., Cornilleau-Wehrlin, N., El-Lemdani Mazouz, F., Schriver, D., Meredith, N. P., and Fazakerley, A.: Wave-particle interactions in the equatorial source region of whistler-mode emissions, J. Geophys. Res.-Space, 115, A00F16, doi:10.1029/2009JA015218, 2010.

Shprits, Y. Y., Thorne, R. M., Horne, R. B., and Summers, D.: Bounce-averaged diffusion coefficients for fieldaligned chorus waves, J. Geophys. Res.-Space, 111, A10225, doi:10.1029/2006JA011725, 2006.

Shprits, Y. Y., Subbotin, D. A., Meredith, N. P., and Elkington, S. R.: Review of modeling of losses and sources of relativistic electrons in the outer radiation belt II: Local acceleration and loss, J. Atmos. Sol.-Terr. Phy., 70, 1694-1713, doi:10.1016/j.jastp.2008.06.014, 2008.

Summers, D.: Quasi-linear diffusion coefficients for fieldaligned electromagnetic waves with applications to the magnetosphere, J. Geophys. Res.-Space, 110, A08213, doi:10.1029/2005JA011159, 2005.

Summers, D. and Ni, B.: Effects of latitudinal distributions of particle density and wave power on cyclotron resonant diffusion rates of radiation belt electrons, Earth, Planets, and Space, 60, 763$771,2008$.

Summers, D., Ni, B., and Meredith, N. P.: Timescales for radiation belt electron acceleration and loss due to resonant wave-particle interactions: 2. Evaluation for VLF chorus, ELF hiss, and electromagnetic ion cyclotron waves, J. Geophys. Res., 112, A04207, doi:10.1029/2006JA011993, 2007.

Summers, D., Tang, R., and Omura, Y.: Effects of nonlinear wave growth on extreme radiation belt electron fluxes, J. Geophys. Res.-Space, 116, A10226, doi:10.1029/2011JA016602, 2011.

Tao, X., Bortnik, J., Thorne, R. M., Albert, J. M., and Li, W.: Effects of amplitude modulation on nonlinear interactions between electrons and chorus waves, Geophys. Res. Lett., 39, L06102, doi:10.1029/2012GL051202, 2012.

Thorne, R. M.: Energetic radiation belt electron precipitation - A natural depletion mechanism for stratospheric ozone, Science, 195, 287-289, doi:10.1126/science.195.4275.287, 1977. 
Thorne, R. M.: Radiation belt dynamics: The importance of wave-particle interactions, Geophys. Res. Lett., 37, L22107, doi:10.1029/2010GL044990, 2010.

Trakhtengerts, V. Y.: A generation mechanism for chorus emission, Ann. Geophys., 17, 95-100, doi:10.1007/s00585-999-0095-4, 1999.

Trakhtengertz, V. Y.: Stationary States of the Earth's Outer Radiation Zone, Geomagn. Aeronom., 6, 638-45, 1966.

Tsurutani, B. T., Falkowski, B. J., Verkhoglyadova, O. P., Pickett, J. S., SantolíK, O., and Lakhina, G. S.: Dayside ELF electromagnetic wave survey: A Polar statistical study of chorus and hiss, J. Geophys. Res.-Space, 117, A00L12, doi:10.1029/2011JA017180, 2012.

Wang, C., Zong, Q., Xiao, F., Su, Z., Wang, Y., and Yue, C.: The relations between magnetospheric chorus and hiss inside and outside the plasmasphere boundary layer: Cluster observation, J. Geophys. Res.-Space, 116, A07221, doi:10.1029/2010JA016240, 2011.

Watt, C. E. J., Rankin, R., and Degeling, A. W.: Whistler mode wave growth and propagation in the prenoon magnetosphere, J. Geophys. Res.-Space, 117, A06205, doi:10.1029/2012JA017765, 2012.
Wilken, B., Axford, W. I., Daglis, I., Daly, P., Guttler, W., Ip, W. H., Korth, A., Kremser, G., Livi, S., Vasyliunas, V. M., Woch, J., Baker, D., Belian, R. D., Blake, J. B., Fennell, J. F., Lyons, L. R., Borg, H., Fritz, T. A., Gliem, F., Rathje, R., Grande, M., Hall, D., Kecsuemety, K., McKenna-Lawlor, S., Mursula, K., Tanskanen, P., Pu, Z., Sandahl, I., Sarris, E. T., Scholer, M., Schulz, M., Sorass, F., and Ullaland, S.: RAPID - The Imaging Energetic Particle Spectrometer on Cluster, Space Sci. Rev., 79, 399-473, doi:10.1023/A:1004994202296, 1997.

Wrenn, G. L., Rodgers, D. J., and Ryden, K. A.: A solar cycle of spacecraft anomalies due to internal charging, Ann. Geophys., 20, 953-956, doi:10.5194/angeo-20-953-2002, 2002.

Zhou, Q., Shi, J., and Xiao, F.: A Three-Dimensional Ray Tracing Study on Whistler-Mode Chorus During Geomagnetic Activities, Plasma Sci. Technol., 13, 440-445, doi:10.1088/10090630/13/4/11, 2011. 